\title{
HYPERCENTRAL INJECTORS IN INFINITE SOLUBLE GROUPS
}

\author{
by M. J. TOMKINSON \\ (Received 15th February 1978)
}

The Carter subgroups of a finite soluble group may be characterised either as the self-normalising nilpotent subgroups or as the nilpotent projectors. Subgroups with properties analogous to both of these have been considered by Newell $(2,3)$ in the class of $\mathfrak{S}_{1}$-groups. The results obtained are necessarily less satisfactory than in the finite case, the subgroups either being almost self-normalising (i.e. having finite index in their normaliser) or having an almost-covering property. Also the subgroups are not necessarily conjugate but lie in finitely many conjugacy classes.

Here we consider the dual concept of nilpotent injectors. A nilpotent subgroup $V$ of a finite soluble group $G$ is a nilpotent injector if, for each subnormal subgroup $S$ of $G, V \cap S$ is a maximal nilpotent subgroup of $S$. These subgroups may also be characterised as the maximal nilpotent subgroups containing the Fitting subgroup. In $\Im_{1}$-groups, maximal hypercentral subgroups containing the hypercentral radical necessarily exist and one might therefore expect the nilpotent injectors to generalise to $\mathfrak{S}_{1}$-groups in a more satisfactory way than the nilpotent projectors.

We shall see that this is in fact the case, proving that in an $\varsigma_{1}$-group the hypercentral injectors coincide with the maximal hypercentral subgroups containing the hypercentral radical and form a unique conjugacy class. The results depend heavily on the fact that $\mathfrak{S}_{1}$-groups are nilpotent-by-abelian-by-finite and so the hypercentral subgroups containing the radical are all finite extensions of the radical. The definition and basic results concerning $\widetilde{S}_{1}$-groups may be found in (4).

The radical $R$ of the $\mathscr{S}_{1}$-group $G$ has a finite series

$$
1=R_{0} \leqslant \cdots \leqslant R_{n}=R
$$

of normal subgroups of $G$ such that each factor $R_{i} / R_{i-1}$ is either

(1) torsion-free abelian and rationally irreducible (as a $G$-module)

or (2) an abelian Černikov p-group.

The torsion-free factors in this series are central in $R$. If we define

$$
N=\bigcap\left\{C_{G}\left(R_{i} / R_{i-1}\right) ; R_{i} / R_{i-1} \text { is torsion-free }\right\}
$$

then $N \geqslant R$.

We write $\boldsymbol{\sigma}(R)$ for the set of primes $\left\{p ; R_{i} / R_{i-1}\right.$ is a $p$-group for some $\left.i\right\}$. For each $p \in \approx(R)$, define

$$
N_{p}=\bigcap\left\{C_{N}\left(\Omega_{1}\left(R_{i} / R_{i-1}\right)\right) ; R_{i} / R_{i-1} \text { is a } p \text {-group }\right\} \text {. }
$$

Then $N / N_{p}$ is finite and $N_{p}$ centralises $\Omega_{n+1}\left(R_{i} / R_{i-1}\right) / \Omega_{n}\left(R_{i} / R_{i-1}\right)$. Thus each $p$-factor $R_{i} / R_{i-1}$ is hypercentral in $N_{p}$. 
For each $p \in \boldsymbol{w}(R)$, let $C_{p}=\bigcap_{q \neq p} N_{q}$ and define

$$
M=\bigcap_{p \in \Phi(R)} C_{p}=\bigcap_{p \in \Phi(R)} N_{p} ;
$$

then $N / M$ is finite and $M \leqslant R$.

We relate the maximal hypercentral subgroups of $G$ containing $R$ to certain subgroups of $N / M$. The characterisation given in this result may be compared with a characterisation given by Fischer in the finite case (see (1) VI.7.18).

Theorem 1. $V$ is a maximal hypercentral subgroup of $G$ containing $R$ if and only if $V \leqslant N$ and each Sylow $p$-subgroup of $V / M$ is a Sylow p-subgroup of $C_{p} / M$.

Proof. Let $W_{p} / M$ be a $p$-subgroup of $C_{p} / M$ and $W=\left\langle W_{p} ; p \in w(R)\right\rangle$. If $p$ and $q$ are distinct primes in $\boldsymbol{\sigma}(R)$, then $\left[W_{p}, W_{q}\right] \leqslant C_{p} \cap C_{q}=M$ and so $W_{p} \triangleleft W$. Since each $p^{\prime}$-factor and each torsion-free factor of $M$ is hypercentral in $W_{p}$ it follows that $W_{p}$ is a hypercentral group. Thus $W$ is a product of normal hypercentral subgroups and so is itself hypercentral.

Let $U$ be a hypercentral subgroup of $G$ containing $R$. Then $U / R$ is finite and so $U$ centralises each torsion-free factor $R_{i} / R_{i-1}$; hence $U \leqslant N$. Let $U_{p} / M$ be the Sylow $p$-subgroup of $U / M$; then $U_{p}$ centralises each $p^{\prime}$-factor of $R$. Thus if $p \notin \infty(R)$ then $U_{p} \leqslant M$ i.e. the Sylow $p$-subgroup of $U / M$ is trivial, and if $p \in \varpi(R)$ then $U_{p} \leqslant C_{p}$.

A straightforward argument using the results of these two paragraphs yields the required result.

Theorem 2. The maximal hypercentral subgroups of $G$ containing $R$ are conjugate in $G$.

Proof. Let $V_{1}$ and $V_{2}$ be maximal hypercentral subgroups of $G$ containing $R$. For $i=1,2$, there is a Sylow basis $S_{i}=\left\{S_{p}^{(i)} / M\right\}$ of $N / M$ reducing into $V_{i} / M$. Thus the Sylow $p$-subgroup of $V_{i} / M$ is $\left(V_{i} \cap S_{p}^{(i)}\right) / M=\left(C_{p} \cap S_{p}^{(i)}\right) / M$. Since $N / M$ is a finite soluble group there is an element $x \in N$ such that $x^{-1} S_{p}^{(1)} x=S_{p}^{(2)}$ for each prime $p$. Hence $x^{-1}\left(V_{1} \cap S_{p}^{(1)}\right) x=x^{-1}\left(C_{p} \cap S_{p}^{(1)}\right) x=C_{p} \cap S_{p}^{(2)}=V_{2} \cap S_{p}^{(2)}$ and so $x^{-1} V_{1} x=V_{2}$.

We define injectors in $\varsigma_{1}$-groups to have a rather stronger condition than in the finite definition. A hypercentral subgroup $V$ of the $\widetilde{S}_{1}$-group $G$ is a hypercentral injector of $G$ if, for each ascendant subgroup $A$ of $G, V \cap A$ is a maximal hypercentral subgroup of $A$.

Theorem 3. $V$ is a hypercentral injector of $G$ if and only if $V$ is a maximal hypercentral subgroup of $G$ containing $R$.

Proof. In one direction the result is obvious and so we have to prove that if $V$ is a maximal hypercentral subgroup of $G$ containing $R$ and if $A$ is an ascendant subgroup of $G$, then $V$ is a maximal hypercentral subgroup of $A$. 
Consideration of the subgroups $A \leqslant A R \leqslant G$ shows that we may consider the separate cases $A \geqslant R$ and $A R=G$.

(1) $A \geqslant R$.

The Sylow $p$-subgroup of $V / M$ is a Sylow $p$-subgroup of $C_{p} / M$ and hence the Sylow $p$-subgroup of $(V \cap A) / M$ is a Sylow $p$-subgroup of $\left(C_{p} \cap A\right) / M$, since $C_{p} \cap A$ is a subnormal subgroup of $C_{p}$. Also $C_{p} \cap A=C_{p}(A)$ and so Theorem 1 shows that $V \cap A$ is a maximal hypercentral subgroup of $A$.

(2) $A R=G$.

There is an ascending series

$$
A=A_{0} \triangleleft \cdots \triangleleft A_{\alpha} \triangleleft \cdots \triangleleft A_{y}=G .
$$

If $W$ is a hypercentral subgroup of $A$ containing $V \cap A$, then $\left[W, R \cap A_{\alpha+1}\right] \leqslant R \cap A_{\alpha}$ and so $W R \cap A_{\alpha} \triangleleft W R \cap A_{\alpha+1}$. Thus $W(=W R \cap A)$ is an ascendant subgroup of $W R$ which, being a product of two ascendant hypercentral subgroups, is itself hypercentral. But $W R \geqslant(V \cap A) R=V$ and so $W R=V$. Hence $W=V \cap A$, as required.

In the characterisation given in Theorem 1 , a hypercentral injector $V$ of $G$ corresponds to a subgroup $V / M$ of a finite soluble section of $G$. In the case of polycyclic groups we can determine a different finite soluble section $K / L$ such that the subgroups $V / L$ are the nilpotent injectors of $K / L$. The simple example of $C_{3^{\infty}}$ extended by its involution automorphism shows that no similar result can be obtained for $\widetilde{S}_{1}$-groups.

Letting $R_{i}$ denote the $i$ th term of the upper central series of $R$ and using the residual finiteness of polycyclic groups there is an integer $n$ such that

$$
\left(R^{n} \cap R_{i}\right) /\left(R^{n} \cap R_{i-1}\right)
$$

is torsion-free. We put $L=R^{n}$ so that $R / L$ is finite and $L$ has a central series

$$
1=L \cap R_{0} \leqslant \cdots \leqslant L \cap R_{k}=L
$$

with torsion-free factors. We define

$$
K=\bigcap_{i=1} C_{G}\left(\left(L \cap R_{i}\right) /\left(L \cap R_{i-1}\right)\right)
$$

Theorem 4. Let $G$ be a polycyclic group. With the above notation, $K / L$ is a finite soluble group and $R / L$ is its radical.

$V$ is a maximal nilpotent subgroup of $G$ containing $R$ if and only if $V / L$ is a maximal nilpotent subgroup of $K / L$ containing $R / L$.

Proof. $G / L$ is finite-by-abelian-by-finite and is also residually finite; hence $G / L$ is abelian-by-finite.

Let $F / L$ be the radical of $K / L$. Then $F$ is nilpotent and normal in $G$ and so $F \leqslant R$. Hence the radical of $K / L$ is the finite subgroup $R / L$. But $K / L$ is abelian-by-finite and so if $K / L$ is infinite, then its radical is infinite. Hence $K / L$ is a finite soluble group.

If $V$ is a nilpotent subgroup of $G$ containing $R$, then $V / R$ is finite and hence $V / L$ is finite. Thus $V$ centralises each of the torsion-free factors $\left(L \cap R_{i}\right) /\left(L \cap R_{i-1}\right)$ and so $V \leqslant N$.

If $W / L$ is a nilpotent subgroup of $K / L$, then $W$ is nilpotent. The result now follows easily. 


\section{REFERENCES}

(1) B. HUPPERT, Endliche Gruppen I (Grundlehren der math. Wiss. 134, Springer, 1967).

(2) M. L. NEwELL, Supplements in abelian-by-nilpotent groups, J. London Math. Soc. (2), 11 (1975), 74-80.

(3) M. L. NEWELL, Nilpotent projectors in $\Im_{1}$-groups, Proc. Royal Irish Acad. (Section A) 75 (1975), 107-114.

(4) D. J. S. ROBINSON, Finiteness conditions and generalized soluble groups (Ergebnisse der Math. 62, 63, Springer, 1972).

UNIVERSITY OF GLASGOW 